\title{
Germline mutations in $M E N 1$ are associated with the tumorigenesis of pituitary adenoma associated with meningioma
}

\author{
HAIBO ZHU ${ }^{1}$, YAZHOU MIAO ${ }^{2}$, YUTAO SHEN ${ }^{2}$, JING GUO $^{2}$, WEIYAN XIE ${ }^{2}$, \\ SIDA ZHAO ${ }^{2}$, WEI DONG ${ }^{2}$, YAZHUO ZHANG ${ }^{1-4}$ and CHUZHONG LI ${ }^{1-4}$ \\ ${ }^{1}$ Department of Neurosurgery, Beijing Tiantan Hospital Affiliated to Capital Medical University; ${ }^{2}$ Cell Laboratory, \\ Beijing Neurosurgical Institute, Capital Medical University; ${ }^{3}$ Cell Laboratory, Beijing Institute for \\ Brain Disorders Brain Tumor Center; ${ }^{4}$ Department of Neurosurgery, China National Clinical \\ Research Center for Neurological Diseases, Beijing 100070, P.R. China
}

Received May 13, 2019; Accepted March 9, 2020

DOI: $10.3892 / \mathrm{ol} .2020 .11601$

\begin{abstract}
Pituitary adenoma and meningioma are two of the most common benign tumors in the central nervous system. Pituitary adenoma associated with meningioma (PAM) is a rare disease, the tumorigenesis of which remains unclear. Therefore, the aim of the present study was to investigate the tumorigenesis of PAM. A total of 8,197 patients with pituitary adenoma were analyzed. Furthermore, the clinical data of 57 patients with PAM were compared with patients with multiple endocrine neoplasia 1 (MEN-1) syndrome. Whole exome sequencing (WES) was performed on 23 samples from patients with PAM and the germline mutation was verified by Sanger sequencing. The age of tumor penetrance (age of patients at diagnosis) for PAM was significantly higher than that for patients with MEN-1. Compared with MEN-1 patients, there was a significant association between PAM and female sex $(\mathrm{P}=0.004)$. Clonal analysis and phylogenetic tree construction suggested that the pituitary adenoma and meningioma in PAM don't originate from a common progenitor. WES revealed that 5/23 PAM samples had the recurrent germline mutation $M E N 1$ c.1523G $>$ A; p.G508D, which may be a genetic risk factor for PAM. Compared with patients with sporadic pituitary adenoma, the difference was statistically significant $(\mathrm{P}=0.0004)$. Compared with wild-type $M E N 1$, there was a significant association between the MEN1 mutation and recurrence of pituitary adenoma, young age and
\end{abstract}

Correspondence to: Dr Chuzhong Li, Cell Laboratory, Beijing Neurosurgical Institute, Capital Medical University, 119 South Fourth Ring West Road, Fengtai, Beijing 100070, P.R. China E-mail: lichuzhong@sina.com

Abbreviations: PAM, pituitary adenoma associated with meningioma; SPA, sporadic pituitary adenoma; SM, sporadic meningioma; MEN1, multiple endocrine neoplasia 1; CNS, central nervous system; FFPE, formalin-fixed and paraffin-embedded; $\mathrm{CCF}$, cancer cell fraction

Key words: PAM, MEN1, germline mutation larger diameter of the meningioma. The present study indicated that germline mutations in MEN1 may be associated with the tumorigenesis of PAM.

\section{Introduction}

Pituitary adenomas are relatively common and account for $10-15 \%$ of all intracranial tumors, with an incidence of $~ 3$ cases per 100,000 people globally (1-4). Meningioma is one of the most common intracranial tumor types, with an estimated incidence of 7.86 cases per 100,000 people per year, $~ 80 \%$ of which are benign and World Health Organization classification grade I (5-8). In contrast, pituitary adenoma associated with meningioma (PAM) is a rare disease, with only 33 cases described before 2017 (9). The tumorigenesis of PAM remains unknown and there are currently no known epidemiological or well-characterized genetic associations between meningioma and pituitary adenoma.

MEN1 is located on chromosome 11q13 and is composed of 10 exons that encode a 610 amino acid protein called menin. Mutated forms of MEN1 include nonsense mutations, missense mutations, frameshifts and insertions. Multiple endocrine neoplasia type 1 (MEN-1) syndrome is an autosomal dominant disease caused by germline MEN1 mutations, which lead to the development of multi-focal neoplastic endocrine lesions of the parathyroid glands, endocrine pancreas, duodenum, anterior pituitary, and less commonly, the stomach, adrenal cortex, thymus and lungs (10-12). In addition, various non-endocrine lesions may occur in the skin, central nervous system (CNS) and soft tissues. Asgharian et al (13) reported that meningioma may be a component tumor of MEN-1, and mutations in the MEN1 gene may participate in its pathogenesis. The present study revealed that 5/23 samples from patients with PAM harbored the recurrent germline mutation MEN1 c.1523G>A; p.G508D. However, none of these patients exhibited MEN-1 syndrome-associated symptoms other than the presence of the pituitary adenoma.

\section{Materials and methods}

Patients. There were 8,197 patients with pituitary adenoma admitted to Beijing Tiantan Hospital (Beijing, China) between 
January 1,2005 and December 31,2017 who were retrospectively reviewed. The average age was $44.17 \pm 13.1$ years (ranging from 4-84 years), with 4008 male patients (48.9\%) and 4189 female patients (51.1\%), M/F ratio of 1:1.05). All patients were classified according to preoperative imaging, including hormone, plain and enhanced head magnetic resonance imaging, thin layer skull base computed tomography scanning and three-dimensional reconstruction. Patients who were diagnosed with meningioma and pituitary adenoma simultaneously or successively were included in the present study. The clinical data of patients with MEN-1 were referred by a previous study (14). A total of 119 patients with sporadic pituitary adenoma (SPA) were selected from the 8,197 patients. They were recruited randomly from Beijing Tiantan Hospital between January 1, 2005 and December 31, 2017. Written informed consent was obtained from all of these 119 patients with SPA.

The present study was conducted in accordance with established ethical guidelines as outlined in the Declaration of Helsinki and was approved by the Ethics Committee of Beijing Tiantan Hospital. Written informed consent was obtained from all participants.

Tissue samples and histology. Tissue samples were obtained from the department of neurosurgery, Beijing Tiantan Hospital. Fresh tumor tissue samples were immediately snap-frozen in liquid nitrogen and stored at $-80^{\circ} \mathrm{C}$. A total of 23 patients with PAM (23 pituitary adenoma tissues, nine peripheral blood samples and seven meningioma tissues) were subjected to next generation and Sanger sequencing (Table SI).

Genomic DNA preparation and whole exome sequencing. The genomic DNA from blood and formalin-fixed and paraffin-embedded (FFPE) samples was extracted using the DNeasy blood and tissue kit (Qiagen, Inc.) and the GeneRead DNA FFPE kit (Qiagen, Inc.), respectively, according to the manufacturer's protocol. DNA degradation and contamination were assessed using a $1 \%$ agarose gel and the concentration was measured using a Qubit ${ }^{\circledR}$ DNA Assay kit and a Qubit ${ }^{\circledR} 2.0$ Flurometer (both Thermo Fisher Scientific, Inc.), according to the manufacturer's protocols. For whole exome sequencing (WES), the genomic DNA from 23 patients with PAM, including 23 pituitary adenoma samples, nine peripheral blood samples and seven meningioma tissues, were fragmented using a S220 Focused-ultrasonicator (Covaris, Inc.), with resultant library fragments $180-280 \mathrm{bp}$ in length, according to the manufacturer's protocol.

Sequence data quality control. The original fluorescence image files obtained from the HiSeq X10 platform were transformed to short reads by base calling and recorded in FASTQ format, which contained sequence information and corresponding sequencing quality information. After excluding reads containing adapter contamination and low-quality/unrecognizable nucleotides, clean data were used for downstream analyses. Additionally, the total read number, sequencing error rate, percentage of reads with average quality $>20$ or $>30$, and GC content distribution were calculated.

Read mapping and somatic genetic alteration detection. Valid sequencing data were mapped onto the reference human genome (UCSC hg19) using Burrows-Wheeler Aligner software to obtain the original mapping results in BAM format (15). SAMtools version 1.0 (16), Picard version 1.111(1901) (broadinstitute.github.io/picard) and GATK version 3.1-1-g07a4bf8 (17) were subsequently used to process the BAM files, and to perform duplicate marking, local realignment and base quality recalibration to generate the final BAM files for computing the sequence coverage and depth.

The tumor and blood samples were subjected to WES and the mean coverage was $\sim 500 \mathrm{x}$ for tumor samples and $\sim 100 \mathrm{x}$ for matched blood samples. MuTect version 1.1.4 and Strelka version 1.0.1 were used to identify somatic single nucleotide variations (SNVs) and small insertions and deletions (InDels), respectively, in the paired tumor and normal blood samples $(18,19)$.

Cloning frequency analysis. Pituitary adenoma, meningioma and blood samples from one patient with PAM were used for cloning frequency analysis and phylogenetic tree construction. The cloning evolutionary analysis was based on the purity of the tumor samples, the change in the copy number and the allele frequency of the somatic mutation, and was used to calculate the cancer cell fraction (CCF) and to identify tumor sample subgroups. PyClone is a hierarchical Bayesian model that infers the cellular prevalence of each variant (the proportion of tumor cells in a sample that contains the variant), and clusters variants based on the covariance of these prevalence estimates across multiple samples obtained from the same patient (20). PyClone software (version 13.0) (20) was used to perform clonality and evolutionary analyses based on the information obtained on genetic somatic mutations and copy number variations (CNVs) of the pituitary tumor and meningioma. For each variant, the $\mathrm{CCF}$ was defined as variant allele fraction $(\mathrm{VAF})=\mathrm{p} \times \mathrm{CCF} /[\mathrm{CT} \times \mathrm{p}+\mathrm{CN}(1-\mathrm{p})]$, where $\mathrm{CT}$ is the copy number of the tumor sample, $\mathrm{CN}$ is the copy number of the matched normal blood sample and $p$ is the tumor purity. Clonal status was defined according to the confidence interval of the CCF. For each somatic mutation, the VAF was calculated using the number of reads supporting the variant allele (Rmut) and the number of reads supporting the reference allele [Rnorm; namely, VAF=Rmut/(Rmut + Rnorm)].

Phylogenetic tree construction. Phylogenetic trees for the multiple tumor samples obtained from the same patient were constructed manually. Only somatic non-synonymous mutations were considered and point mutations and InDels were incorporated. Somatic non-synonymous alterations that were shared in both pituitary adenoma and meningioma in the same case were counted towards the trunk; likewise, private mutations, those mutations only present in either the pituitary adenoma or meningioma, were counted towards the branches. The length of the trunk and branches were proportional to the number of somatic non-synonymous mutations.

Sanger validation. MEN1 candidate point mutations identified from exome sequencing were validated using Sanger sequencing. Genomic sequences around candidate mutations were obtained from the National Center for Biotechnology Information. Primers were designed using Primer 3 software (version 0.4.0; bioinfo.ut.ee/primer3-0.4.0) and were 
A

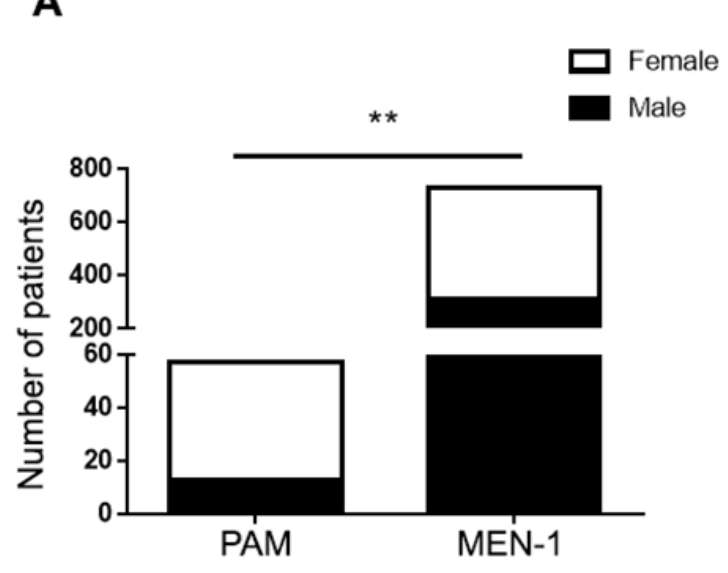

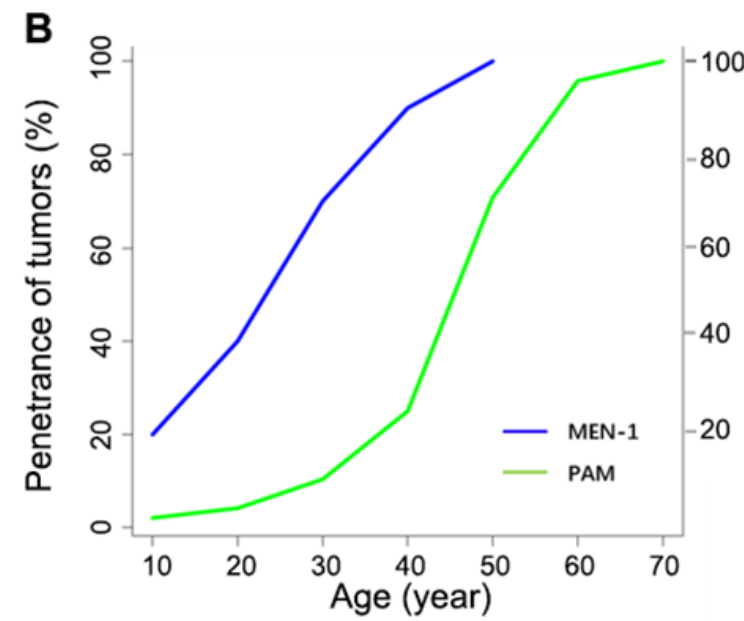

Figure 1. Analysis of the clinical variables of patients with PAM and MEN-1 syndrome. (A) Sex differences $\left(\chi^{2}\right.$ test) and (B) age of tumor penetrance in patients with PAM and MEN-1 syndrome. ${ }^{* *} \mathrm{P}<0.01$. MEN-1, multiple endocrine neoplasia 1; PAM, pituitary adenoma associated with meningioma; SM, sporadic meningioma; SPA, sporadic pituitary adenoma.

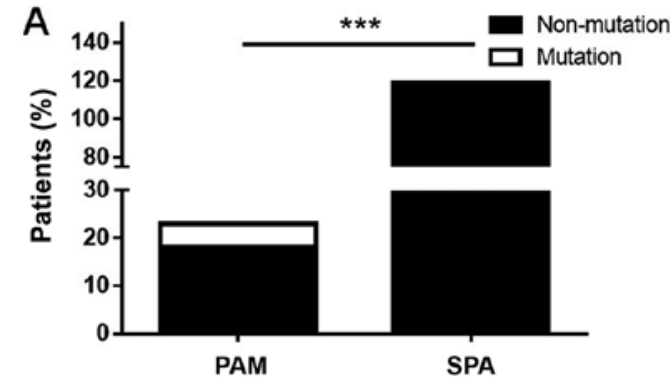

C

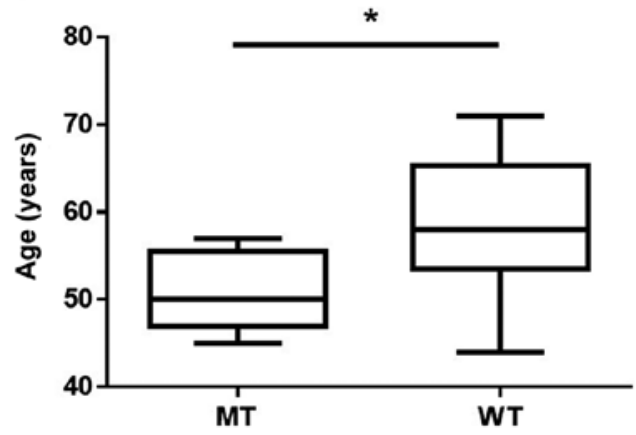

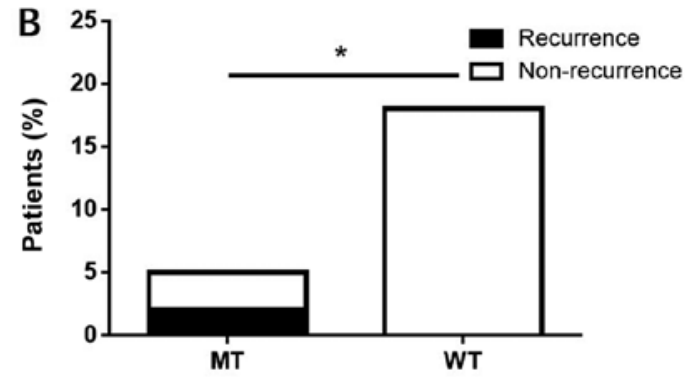

D

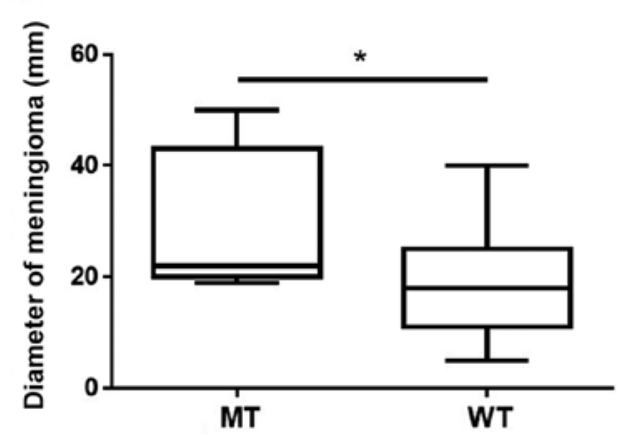

Figure 2. Germline mutation of MEN1 (c.1523G>A; p.G508D) may serve as a genetic predisposition factor for PAM. (A) Germline mutation of MEN1 (c.1523G $>$ A; p.G508D) in patients with PAM and SPA ( $\chi^{2}$ test). (B) Recurrence rate of pituitary adenoma in patients with PAM with WT and MT MEN1 (Fisher's exact test). (C) Age difference between patients with PAM with WT and MT MEN1 (unpaired Student's t-test). (D) Difference in meningioma diameter between WT and MT MEN1 in PAM (unpaired Student's t-test). ${ }^{*} \mathrm{P}<0.05,{ }^{* * * *} \mathrm{P}<0.001$. MEN1, multiple endocrine neoplasia 1; MT, mutant type, 5 cases; PAM, pituitary adenoma associated with meningioma; SPA, sporadic pituitary adenoma; WT, wild type, 18 cases.

as follows: $M E N 1$, forward 5'-CCGTGAGTTGCAGCTTGA TG-3' and reverse 5'-CAACCTTGCTCTCACCTTGC-3'.

The DNA samples obtained from the 23 patients were subjected to PCR to validate the candidate mutation MENI c.1523G $>$ A; p.G508D. The PCR mixture contained $25 \mu 12 \mathrm{X}$ TSINGKE Master mix (blue) (TSINGKE) (http://www.tsingke. net/shop/), $22 \mu \mathrm{l}$ double distilled $\mathrm{H}_{2} \mathrm{O}, 1 \mu \mathrm{l} 10 \mu \mathrm{M}$ forward and reverse primer, and $1 \mu \mathrm{l}$ template DNA at $10 \mathrm{ng} / \mu \mathrm{l}$, resulting in a final volume of $50 \mu \mathrm{l}$. The thermocycling conditions were as follows: $5 \mathrm{~min}$ at $94^{\circ} \mathrm{C} ; 10$ cycles of $30 \mathrm{sec}$ at $94^{\circ} \mathrm{C}, 30 \mathrm{sec}$ at $61^{\circ} \mathrm{C}$ and $1 \mathrm{~min}$ at $72^{\circ} \mathrm{C} ; 30$ cycles of $30 \mathrm{sec}$ at $94^{\circ} \mathrm{C}, 30 \mathrm{sec}$ at $56^{\circ} \mathrm{C}$ and 1 min at $72^{\circ} \mathrm{C}$; and 3 min at $72^{\circ} \mathrm{C}$.
Following amplification of the DNA sequences, Sanger sequencing was performed on all 23 samples according to the TSINGKE protocol. The sequencing traces were visualized using Codon Code Aligner software version 6.0.2 (TSINGKE) to confirm the presence of candidate mutations.

Statistical analysis. Statistical analyses were performed using SPSS software (v20.0; IBM Corp.). Statistical comparisons of MEN1 mutation status were performed using Fisher's exact test. The $\chi^{2}$ test was used to analyze ordinal variables and unpaired Student's t-test was used to test continuous data. $\mathrm{P}<0.05$ was considered to indicate a statistically significant difference. 
A

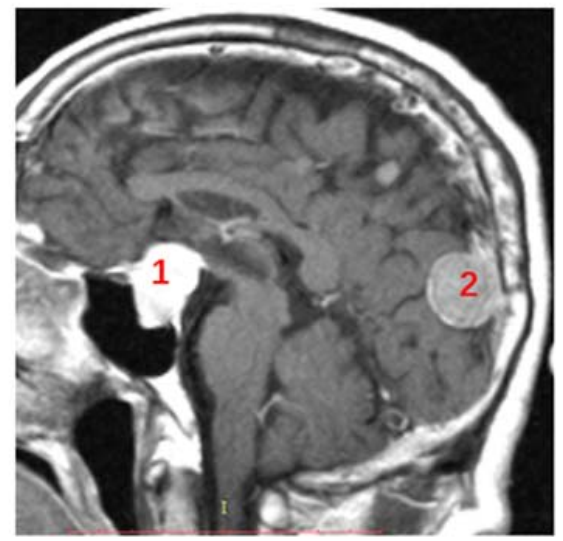

C

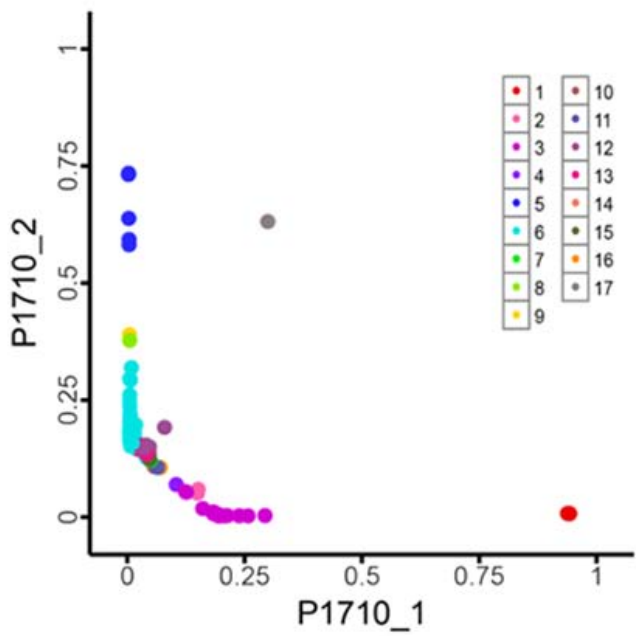

B

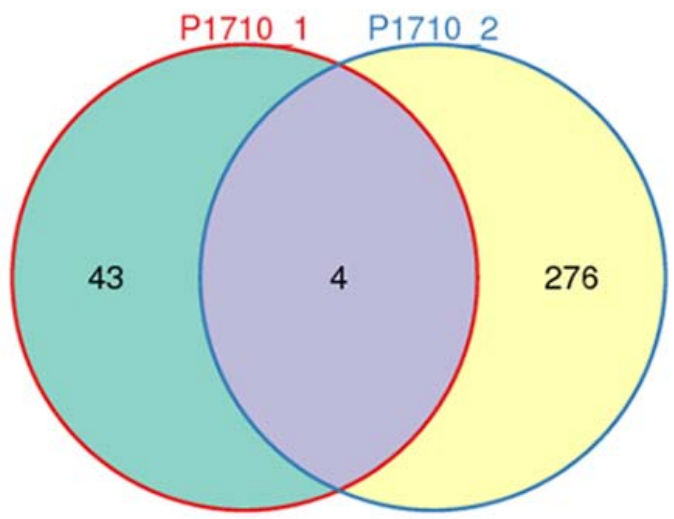

D

Figure 3. Pituitary adenoma and meningioma in PAM do not originate from a common progenitor. (A) Magnetic resonance imaging revealed the tumo sites in patients: 1, pituitary adenoma; 2, meningioma. (B) A Venn diagram was used to examine the relationship of somatic mutations between the pituitary adenoma (green circle) and meningioma (yellow circle) in a patient with PAM. The different number represents the number of somatic mutations for corresponding samples and the overlapped regions are ubiquitous somatic mutations between two concurrent tumor components in the same patient with PAM. (C) Two-dimensional scatter plots show the CCF of the mutations in the pituitary adenoma and meningioma components of tumors. The clusters off the axes indicate mutations in both of tumor components. The clusters on the axes reveal mutations in either pituitary adenoma or meningioma components. (D) The inferred phylogenetic trees of the patient with PAM. Fraction of ubiquitous non-synonymous somatic mutations (trunk; gray bar) and unique non-synonymous somatic mutations (branch; blue bar for pituitary adenoma and pink bar for meningioma) reveal the relationship between the two tumor samples obtained from a patient with PAM. CCF, cancer cell fraction; PAM, pituitary adenoma associated with meningioma.

\section{Results}

Clinical features. In the present study, $57(0.7 \%)$ patients met the criteria for PAM. The average age was $54.2 \pm 9.8$ years (range 20-71 years), with 44 female and 13 male patients. Compared with patients with MEN-1 (734 cases stated in the previous study) (14), there was a significant association between PAM and the female sex ( $\mathrm{P}=0.004$; Fig. 1A). The age of tumor penetrance is the age of patients at diagnosis. The age of tumor penetrance for PAM was significantly higher than for patients with MEN-1 (14). The penetrance rate of MEN-1 syndrome-related tumors before the age of 40 was $94 \%$, but only $25 \%$ for PAM (Fig. 1B). Only one patient out of the 119 patients with SPA sequenced on the HiSeq X10 platform harbored the hotspot site (MEN1 c.1523G>A; p.G508D). The difference was statistically significant ( $\mathrm{P}=0.0004$; Fig. 2A). Compared with wild-type $M E N 1$, patients with a MEN1 mutation were more likely to suffer from a recurrence of pituitary adenoma, develop a pituitary adenoma at a younger age and suffer from a larger diameter of the meningioma $(\mathrm{P}<0.05$; Fig. 2B-D).

Pituitary adenoma and meningioma components do not share a common clone origin in PAM. Pituitary adenoma and meningioma samples obtained from one patient with PAM, indicated in Fig. 3A, were subjected to WES. The average depths of WES were 500x for the tumor samples and 100x for the peripheral blood samples. There were 47 somatic SNVs in the pituitary adenoma and 280 somatic SNVs in the meningioma. To explore the relationship between pituitary adenoma and meningioma components in the same patient, somatic SNVs were classified 


\begin{tabular}{|c|c|}
\hline $\begin{array}{l}\text { 8,197 pitui } \\
\text { December }\end{array}$ & $\begin{array}{l}\text { tary adenoma patients from January } 1,2005 \text { to } \\
31,2017\end{array}$ \\
\hline 57 patient & $s$ met the criteria for PAM \\
\hline $\begin{array}{l}23 \text { patients } \\
\text { blood) }\end{array}$ & s with PAM had samples (23 PAs, 7 meningiomas, 9 \\
\hline WES: Tissu & e (FFPE) 500X, Blood 100X \\
\hline & Filtered by MEN1 \\
\hline Total MEN & 1 SNP/Indels: 131 \\
\hline & Nonsynonymous mutation in coding sequence \\
\hline Non-syno & nymous MEN1 SNP/Indels: 46 \\
\hline & Filtered by $1-\mathrm{k}$ genomic and ExAC \\
\hline rs3758042 & 228: $5 / 23$ patients \\
\hline & Compared with single PA (1/119) \\
\hline$P<0.05, s$ & significantly different \\
\hline & Sanger sequencing \\
\hline Confirmati & ion: MEN1 c. $1523 G>A: p . G 508 D$ \\
\hline
\end{tabular}

Figure 4. Flowchart of gene screening in PAM. FFPE, formalin-fixed and paraffin-embedded; Indels, Insertions/deletions; MEN1, multiple endocrine neoplasia 1; PAM, pituitary adenoma associated with meningioma; PAs, pituitary adenomas; SNP, single nucleotide polymorphism; WES, whole exome sequencing.

as ubiquitous (present in both tumor components) or private (only present in a single tumor). Only four somatic SNVs were present in both the pituitary adenoma and meningioma samples (Fig. 3B). These data indicated that it is likely that SNVs in pituitary adenoma and meningioma components accumulated independently. In addition, clonal analyses suggested different mutation clusters for the patient with PAM after adjusting for $\mathrm{CCF}$ in the samples using PyClone (Fig. 3C). To further explore the clonal structure of PAM, phylogenetic trees were constructed using somatic non-synonymous SNVs. The results revealed a short trunk length, which suggested that PAM may be of polyclonal origin (Fig. 3D).

The aforementioned results, obtained by analyzing somatic mutations, clonal analyses and phylogenetic tree construction, implied that pituitary adenoma and PAM do not originate from a common progenitor.

Gene screening for PAM predisposition. SNVs/InDels in a single tumor sample lacking a matched control were obtained through alignment with the UCSC Human Genome Reference hg19 using SAMtools. A total of 131 MEN1 SNVs/InDels were identified in all the samples. The 46 variants that were non-synonymous mutations in protein-coding regions were selected for further investigation. In addition, polymorphisms of somatic SNVs and InDels referenced in the 1000 Genomes Project (21) or Exome Aggregation Consortium (22) with a minor allele frequency $>1 \%$ were removed. The hotspot site rs375804228 (MEN1 c.1523G>A; p.G508D), which was found in 5 of 23 patients with PAM, was further investigated (Fig. 4). However, the patients did not have MEN-1 syndrome-related symptoms, and the imaging examination and laboratory investigations were not indicative of MEN-1 syndrome. Five patients with MEN1 germline mutation (MEN1 c.1523G>A; p.G508D) were analyzed using Sanger sequencing. Two patients had a pituitary adenoma sample and a matched blood sample, one patient had a meningioma sample and a matched blood sample, and one patient had only pituitary adenoma sample. MEN1 germline mutations (MEN1 c.1523G>A; p.G508D) were detected in these samples. Patient 2 had a loss of heterozygosity on MEN1 c.1523G $>$ A; p.G508D in the pituitary adenoma sample, which may contribute to tumorigenesis in PAM (Fig. 5).

\section{Discussion}

Pituitary adenoma and meningioma are the most common benign tumors in the CNS (9). A total of 57 novel PAM cases were identified from 8,197 pituitary adenoma cases in the present study. Khandwala et al (23) reported that the levels of growth hormone $(\mathrm{GH})$ and insulin-like growth factor 1 may play an important role in the tumorigenesis of PAM. However, in the present study, there were only six patients with GH pituitary adenoma. There may be a common genetic mechanism leading to PAM, particularly mutations of tumor suppressor genes. The present study used somatic mutation and clonal 


\section{Patient 1}
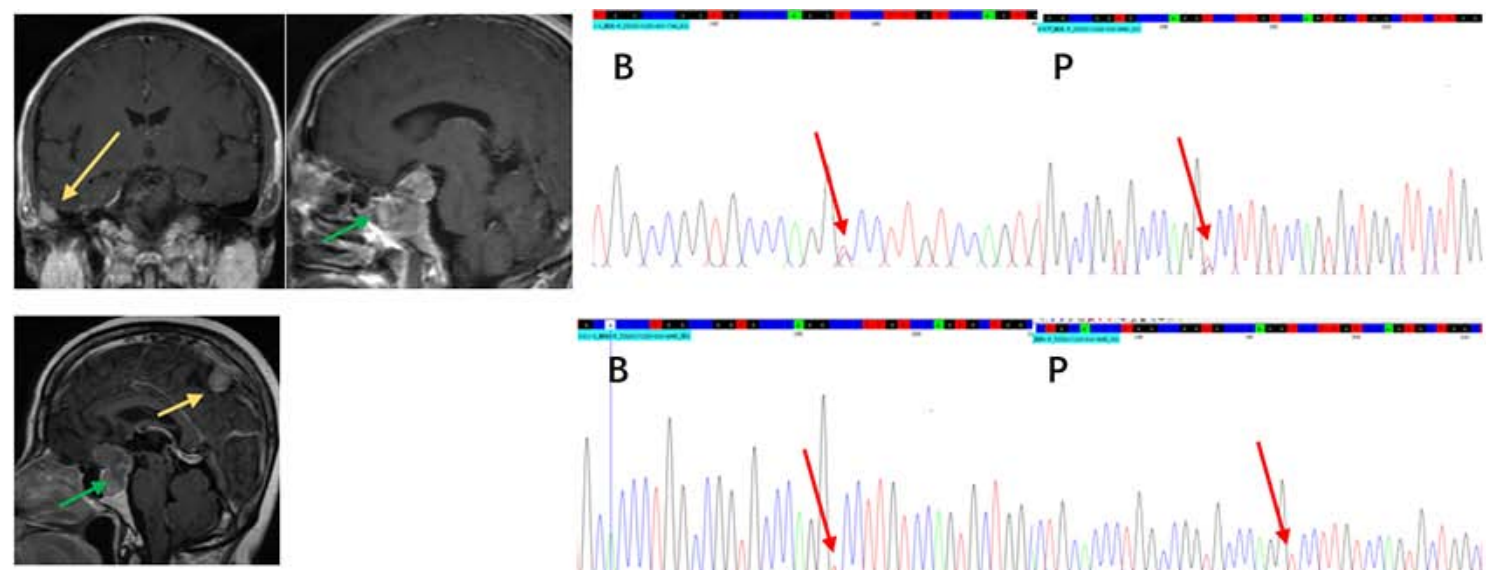

Patient 2
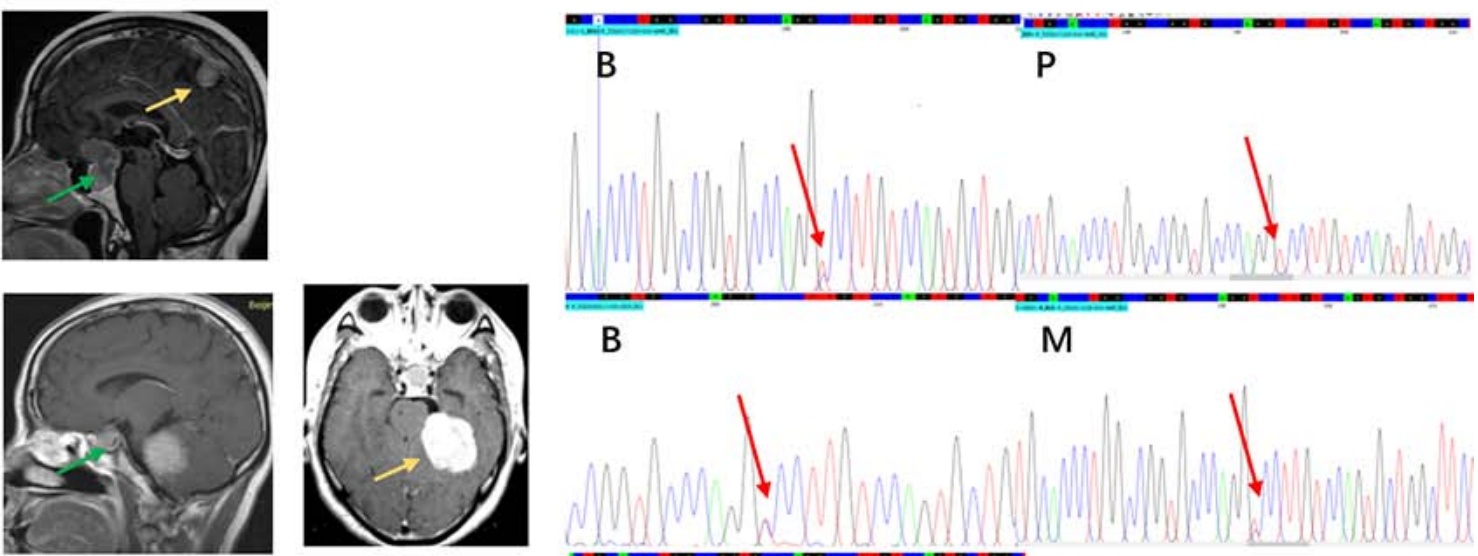

B

M

Patient 3
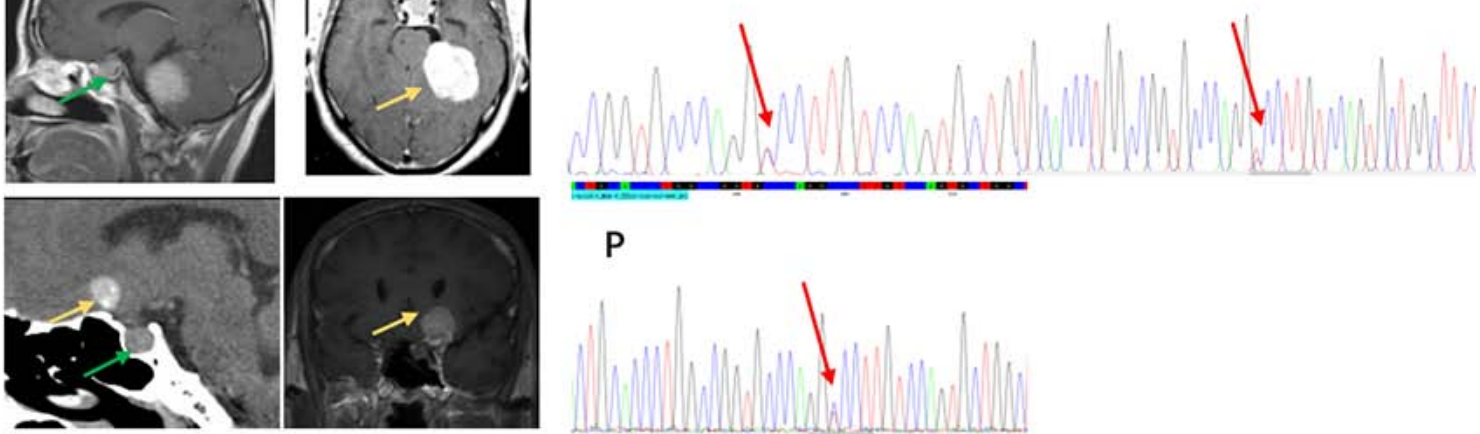

$\mathrm{P}$

Patient 4

Patient 5
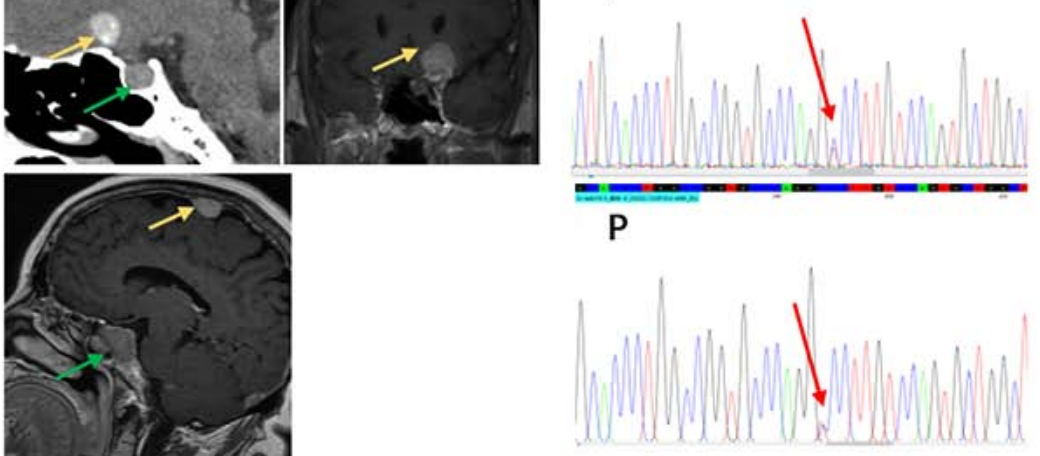

Figure 5. Imaging and Sanger sequencing of 5 patients with PAM carrying the germline mutation $M E N 1$ c.1523G $>$ A; p.G508D. Red arrows indicate mutated bases, yellow arrows indicate meningioma, green arrow indicate pituitary adenoma. Patient 1 had pituitary adenoma and right temporal meningioma and possessed the germline mutation MEN1 c.1523G>A; p.G508D. Patient 2 had pituitary adenoma and convex meningioma had loss of heterozygosity on MEN1 c.1523G>A; p.G508D. Patient 3 had pituitary adenoma and left cerebellopontine angle meningioma and possessed the germline mutation $M E N 1$ c.1523G $>$ A; p.G508D. Patient 4 had pituitary adenoma and tuberculum sellae meningioma and possessed the germline mutation $M E N 1$ c.1523G $>$ A; p.G508D. Patient 5 had pituitary adenoma and convex meningioma and possessed the germline mutation MEN1 c.1523G>A; p.G508D. B, blood; P, pituitary adenoma; M, meningioma; MEN1, multiple endocrine neoplasia 1; PAM, pituitary adenoma associated with meningioma.

analyses to reveal that, in PAM, the pituitary adenoma and meningioma were not found to originate from a common progenitor. Therefore, the most likely explanation for the development of PAM is a genetic predisposition.

The present study revealed that 5/23 patients with PAM had the same germline mutation in MEN1 (c.1523G >A; p.G508D), compared with 1/119 patients with SPA. Therefore, the germline mutation may be a genetic risk factor for the development of PAM. However, the results of the present study showed that there were significant differences between PAM and MEN-1 syndrome (Table SII). Both sexes are equally affected, with no geographical, racial or ethnic differences (13). Mutated forms of MEN1 include nonsense mutations, missense mutations, frameshifts and insertions. In addition, previous studies revealed that certain endocrine tumors, as well as non-endocrine tumors, such as skin tumors, lipomas and carcinoids, are associated with patients with MEN-1 syndrome $(24,25)$. CNS tumors, including ependymomas, schwannomas and meningiomas, have rarely been reported in patients with MEN-1 or MEN-1 variant syndromes $(13,26)$. For patients with PAM, related tumors include pituitary adenoma and meningioma, and the age of tumor penetrance for PAM is significantly higher than that for patients with MEN-1 syndrome (14). In the present study, the penetrance rate of tumors related to MEN-1 syndrome was $94 \%$ in patients $<40$ years old, but was only $25 \%$ for patients with PAM. Furthermore, compared with patients with MEN-1 syndrome, there was a significant association between PAM and the female sex. Additionally, the mutated form of PAM only included a missense mutation.

The present study has some limitations. This is a retrospective study and the incidence of PAM is low. The number of meningioma specimens from patients with PAM collected for exome sequencing is also low. Samples will continue to be collected for further study. More experiments and data may be needed to support this study in the future. 
The present study revealed that the pituitary adenoma and meningioma tissues obtained from one patient with PAM did not originate from a common progenitor. The germline mutation MEN1 (c.1523G>A; p.G508D) may serve as a genetic predisposition for PAM. Compared with wild-type MEN1, there was a significant association between MENI mutation and recurrence of pituitary adenoma, young age and larger diameter of the meningioma.

\section{Acknowledgements}

The authors would like to thank Dr Chengcheng Wang (Etiology Laboratory, National Cancer Center/Cancer Hospital Chinese Academy of Medical Sciences) for experimental guidance, Dr Zhexuan Li (Epidemiological Laboratory, Beijing Cancer Hospital/Beijing Institute For Cancer Research) for support with the statistical analysis and Mr. Lei Gong and Mrs. Hongyun Wang (Cell Laboratory, Beijing Neurosurgical Institute) for support with technique.

\section{Funding}

The present study was supported by grants from the National High Technology Research and Development Program of China 863 Program (grant no. 2014AA020610), the National Natural Science Foundation of China (grant no. 81771489), the Beijing Municipal Science \& Technology Commission (grant no. Z171100000117002) and the China National Key Research and Development Program (grant no. 2017YFC0908300).

\section{Availability of data and materials}

All data generated or analyzed during this study are included in this published article.

\section{Authors' contributions}

HBZ, CZL and YZZ designed the study. HBZ, YZM, JG and YTS collected the samples and clinical data. HBZ, SDZ and WYX performed the experiments. HBZ and CZL contributed to manuscript writing and submission. HBZ, YZM, JG and WD performed the statistical analyses and figure formatting. CZL and YZZ participated in the funding application and management of the project. All authors read and approved the final manuscript.

\section{Ethics approval and consent to participate}

This study was approved by the Institutional Research Board of Beijing Tiantan Hospital, Capital Medical University. Written informed consent was obtained from all participants.

\section{Patient consent for publication}

Not applicable.

\section{Competing interests}

The authors declare that they have no competing interests.

\section{References}

1. McDowell BD, Wallace RB, Carnahan RM, Chrischilles EA, Lynch CF and Schlechte JA: Demographic differences in incidence for pituitary adenoma. Pituitary 14: 23-30, 2011.

2. Lecoq AL, Kamenický P, Guiochon-Mantel A and Chanson P: Genetic mutations in sporadic pituitary adenomas-what to screen for? Nat Rev Endocrinol 11: 43-54, 2015.

3. Ezzat S, Asa SL, Couldwell WT, Barr CE, Dodge WE, Vance ML and McCutcheon IE: The prevalence of pituitary adenomas: A systematic review. Cancer 101: 613-619, 2004.

4. Melmed S: Pathogenesis of pituitary tumors. Nat Rev Endocrinol 7: 257-266, 2011.

5. Amirjamshidi A, Mortazavi SA, Shirani M, Saeedinia S and Hanif $\mathrm{H}$ : 'Coexisting pituitary adenoma and suprasellar meningioma-a coincidence or causation effect: Report of two cases and review of the literature'. J Surg Case Rep 2017: rjx039, 2017.

6. Ruiz-Juretschke F, Iza B, Scola-Pliego E, Poletti D and Salinero E: Coincidental pituitary adenoma and planum sphenoidale meningioma mimicking a single tumor. Endocrinol Nutr 62: 292-294, 2015.

7. Bondy M and Ligon BL: Epidemiology and etiology of intracranial meningiomas: A review. J Neurooncol 29: 197-205, 1996.

8. Curto L, Squadrito S, Almoto B, Longo M, Granata F, Salpietro F, Torre ML, Marini F, Trimarchi F and Cannavo S: MRI finding of simultaneous coexistence of growth hormone-secreting pituitary adenoma with intracranial meningioma and carotid artery aneurysms: Report of a case. Pituitary 10: 299-305, 2007.

9. Herrero-RuizA,Villanueva-AlvaradoHS, Corrales-Hernandez JJ, Higueruela-Minguez C, Feito-Perez J and Recio-Cordova JM: Coexistence of GH-producing pituitary macroadenoma and meningioma in a patient with multiple endocrine neoplasia type 1 with hyperglycemia and ketosis as first clinical sign. Case Rep Endocrinol 2017: 2390797, 2017.

10. Concolino P, Costella A and Capoluongo E: Multiple endocrine neoplasia type 1 (MEN1): An update of 208 new germline variants reported in the last nine years. Cancer Genet 209: 36-41, 2016.

11. Agarwal SK: The future: Genetics advances in MEN1 therapeutic approaches and management strategies. Endocr Relat Cancer 24: T119-T134, 2017.

12. Weber F and Mulligan LM: Happy 20th anniversary MEN1: From positional cloning to gene function restoration. Endocr Relat Cancer 24: E7-E11, 2017.

13. Asgharian B, Chen YJ, Patronas NJ, Peghini PL, Reynolds JC, Vortmeyer A, Zhuang Z, Venzon DJ, Gibril F and Jensen RT: Meningiomas may be a component tumor of multiple endocrine neoplasia type 1. Clin Cancer Res 10: 869-880, 2004.

14. Goudet P, Bonithon-Kopp C, Murat A, Ruszniewski P, Niccoli P, Ménégaux F, Chabrier G, Borson-Chazot F, Tabarin A, Bouchard P, et al: Gender-related differences in MEN1 lesion occurrence and diagnosis: A cohort study of 734 cases from the groupe d'etude des tumeurs endocrines. Eur J Endocrinol 165: 97-105, 2011.

15. Li H and Durbin R: Fast and accurate long-read alignment with Burrows-Wheeler transform. Bioinformatics 26: 589-595, 2010.

16. Li H, Handsaker B, Wysoker A, Fennell T, Ruan J, Homer N, Marth G, Abecasis G and Durbin R; 1000 Genome Project Data Processing Subgroup: The Sequence Alignment/Map format and SAMtools. Bioinformatics 25: 2078-2079, 2009.

17. DePristo MA, Banks E, Poplin R, Garimella KV, Maguire JR, Hartl C, Philippakis AA, del Angel G, Rivas MA, Hanna M, et al: A framework for variation discovery and genotyping using next-generation DNA sequencing data. Nat Genet 43: 491-498, 2011.

18. Cibulskis K, Lawrence MS, Carter SL, Sivachenko A, Jaffe D, Sougnez C, Gabriel S, Meyerson M, Lander ES and Getz G: Sensitive detection of somatic point mutations in impure and heterogeneous cancer samples. Nat Biotechnol 31: 213-219, 2013.

19. Saunders CT, Wong WS, Swamy S, Becq J, Murray LJ and Cheetham RK: Strelka: Accurate somatic small-variant calling from sequenced tumor-normal sample pairs. Bioinformatics 28: 1811-1817, 2012

20. Roth A, Khattra J, Yap D, Wan A, Laks E, Biele J, Ha G, Aparicio S, Bouchard-Côté A and Shah SP: PyClone: Statistical inference of clonal population structure in cancer. Nat Methods 11: 396-398, 2014.

21. 1000 Genomes Project Consortium, Abecasis GR, Auton A, Brooks LD, DePristo MA, Durbin RM, Handsaker RE, Kang HM, Marth GT and McVean GA: An integrated map of genetic variation from 1,092 human genomes. Nature 491: 56-65, 2012. 
22. Lek M, Karczewski KJ, Minikel EV, Samocha KE, Banks E, Fennell T, O'Donnell-Luria AH, Ware JS, Hill AJ, Cummings BB, et al: Analysis of protein-coding genetic variation in 60,706 humans. Nature 536: 285-291, 2016.

23. Khandwala HM, McCutcheon IE, Flyvbjerg A and Friend KE: The effects of insulin-like growth factors on tumorigenesis and neoplastic growth. Endocr Rev 21: 215-244, 2000.

24. Ito T, Igarashi H, Uehara H, Berna MJ and Jensen RT: Causes of death and prognostic factors in multiple endocrine neoplasia type 1: A prospective study: Comparison of $106 \mathrm{MEN1/Zollinger-Ellison}$ syndrome patients with 1613 literature MEN1 patients with or without pancreatic endocrine tumors. Medicine (Baltimore) 92: 135-181, 2013.
25. Brandi ML, Gagel RF, Angeli A, Bilezikian JP, Beck-Peccoz P, Bordi C, Conte-Devolx B, Falchetti A, Gheri RG, Libroia A, et al: Guidelines for diagnosis and therapy of MEN type 1 and type 2 . J Clin Endocrinol Metab 86: 5658-5671, 2001.

26. Giraud S, Choplin H, Teh BT, Lespinasse J, Jouvet A, Labat-Moleur F, Lenoir G, Hamon B, Hamon P and Calender A: A large multiple endocrine neoplasia type 1 family with clinical expression suggestive of anticipation. J Clin Endocrinol Metab 82: 3487-3492, 1997.

(i) (1) () This work is licensed under a Creative Commons (c) Atribution-NonCommercial-NoDerivatives 4.0 International (CC BY-NC-ND 4.0) License. 